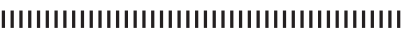

Society Awards 2020

IIIIIIIIIIIIIIIIIIIIIIIIIIIIIIIIIIIIIIIIIII

(on high prospectiveness)

\title{
Studies on the sorption behavior and plant uptake of pesticides in Japanese soils
}

\author{
Yutaka Мотокі* \\ Agricultural Chemicals Inspection Station, Food and Agricultural Materials Inspection Center, \\ 2-772 Suzuki-cho, Kodaira, Tokyo 187-0011, Japan
}

(Accepted July 3, 2020)

\begin{abstract}
To estimate pesticide residue levels in succeeding crops based on those in soils, the relationship between pesticide concentrations in komatsuna (Brassica rapa var. perviridis) and the concentrations extracted sequentially from soils using water and acetone was investigated. The concentrations of many pesticides in komatsuna shoots showed higher positive correlation with water-extractable concentrations $\left(C_{\mathrm{W}}\right)$ than total-extractable concentrations in soils, so that the $C_{\mathrm{W}}$ was available for evaluating the phytoavailability of pesticides in the soil. As a result of examining the dissipation behavior of the $C_{\mathrm{W}}$, the dissipation of the $C_{\mathrm{W}}$ was able to be predicted by considering time-dependent soil sorption, which could be estimated using the sorption coefficients $\left(K_{\mathrm{d}}\right)$ measured by a standard batch method. Furthermore, the present study showed that the properties of soil organic carbon such as black carbon content and the molecular structure of pesticides were important for estimating the $K_{\mathrm{d}}$ values more accurately.

Keywords: plant uptake, phytoavailability, soil aging, time-dependent sorption, organic carbon quality, black carbon.
\end{abstract}

\section{Introduction}

The Japanese Positive List System for pesticides was implemented in 2006. Under this system, the uniform limit was set at $0.01 \mathrm{mg} / \mathrm{kg}$ for pesticides for which maximum residue limits (MRLs) have not been established. ${ }^{1)}$ Since then, some crops that contain pesticides above a concentration of $0.01 \mathrm{mg} / \mathrm{kg}$ cannot be sold or distributed. One reason the limit has been exceeded is that pesticides applied to previous crops and remaining in the soil were detected in succeeding crops. To prevent the production of contaminated crops, a method of estimating pesticide concentrations in succeeding crops before harvest based on the residue concentrations in soils should be developed. The estimation method is available for setting appropriate plant-back intervals, i.e., plant restriction intervals, in a field.

The residue concentrations of pesticides in soils are generally determined by using a vigorous extraction technique from soils

\footnotetext{
* To whom correspondence should be addressed.

E-mail: yutaka_motoki037@famic.go.jp

Published online July 22, 2020
}

(c) BY-NC-ND (c) Pesticide Science Society of Japan 2020. This is an open access article distributed under the Creative Commons AttributionNonCommercial-NoDerivatives 4.0 International (CC BY-NC-ND 4.0) License (https://creativecommons.org/licenses/by-nc-nd/4.0/) with an organic solvent, such as acetone. However, it is assumed that some pesticide residues in soil are eluted into soil water and, subsequently, taken up by plant roots; thus, a vigorous technique that extracts not only pesticides eluted with water but also soilsorbed pesticides is inadequate for assessing the phytoavailability of pesticides in soils. Sugimura et al. ${ }^{2,3)}$ found that the phytotoxicity of pendimethalin and metolachlor in upland soils could be assessed using the concentrations extracted from soils by distilled water. Although they did not measure the residual concentrations of these pesticides in plants, their results imply that quantifying the amount of water-extractable pesticides in soils is important for evaluating the phytoavailability of pesticides in soils.

The water extractability of pesticides in soils increases with decreasing sorption strength to soil; thus, studies on the soil sorption properties of pesticides are crucial to evaluating the phytoavailability of residual pesticides in soils. Nonionic pesticides generally sorb to soil particles via hydrophobic interactions. ${ }^{4}$ ) Therefore, the soil sorption of pesticides increases with increasing organic carbon (OC) content in soil. However, OCnormalized sorption coefficients $\left(K_{\mathrm{oc}}\right)$, which are calculated by dividing the sorption coefficient $\left(K_{\mathrm{d}}\right)$ values by the OC content in soil, of a pesticide are highly variable, depending on the soil type. ${ }^{4-8)}$ Recent sorption studies using foreign soils suggest that the variable physicochemical nature of soil $\mathrm{OC}$ is one reason for the variability of $K_{\mathrm{oc}}$ values. ${ }^{6-8)}$ Furthermore, it has also been re- 


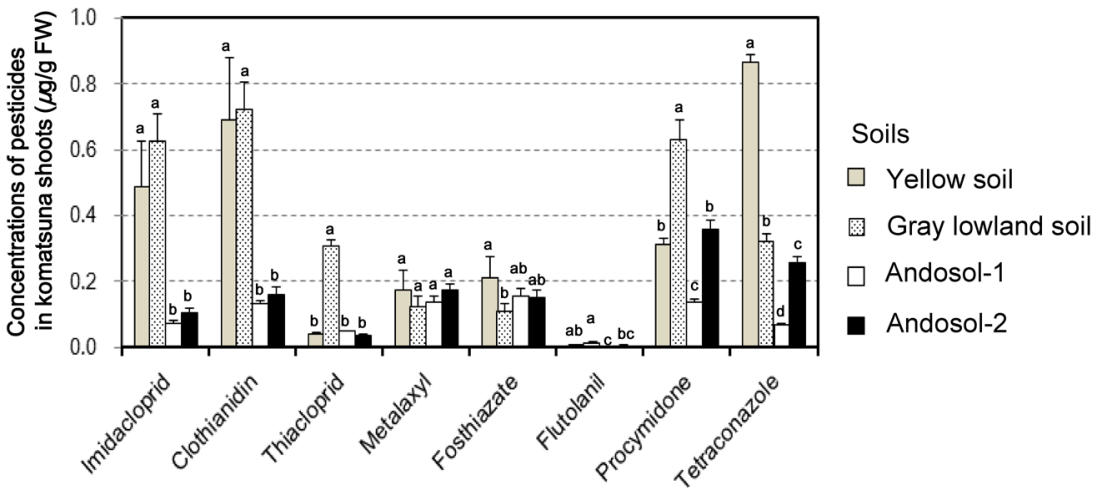

Fig. 1. Pesticide concentrations in komatsuna shoots. Columns with the same letter are not significantly different at $p<0.05$, according to ANOVAprotected Tukey's multiple range testing. Error bars indicate standard deviations $(n=4)$. Reprinted from Ref. 25.

ported that OCs in soils include black carbons (BCs) such as char and soot, which are products of the incomplete combustion of vegetation by wildfires or human activity. ${ }^{9-12)}$ Because BCs strongly sorb organic chemicals including pesticides, the BC content in soils may affect the overall sorption of pesticides in soils. ${ }^{13)}$ However, there are no reports about the effect of the OC quality on the sorption properties of pesticides in Japanese soils, including andosol, which is the major upland soil.

The $K_{\mathrm{d}}$ values are generally determined in accordance with the Organization for Economic Cooperation and Development (OECD) test guidelines, which is the standard batch method. ${ }^{14)}$ In this method, the $K_{\mathrm{d}}$ values are measured by shaking the soil with an aqueous solution for about $24 \mathrm{hr}$ after pesticide application, and daily and weekly time courses of $K_{\mathrm{d}}$ values are not taken into account. However, it is reported that the $K_{\mathrm{d}}$ values of various pesticides increased with time. ${ }^{15-24)}$ Therefore, if the aging effect on the $K_{\mathrm{d}}$ values is not considered, the concentrations of water-extractable pesticides predicted using the $K_{\mathrm{d}}$ values seem to be higher than the actual values.

In order to develop a method for estimating the residue concentrations of pesticides in succeeding crops using those in soils, the present study investigated (i) the relationships between pesticide concentrations in komatsuna (Brassica rapa var. perviridis) and water-extractable concentrations in soils; (ii) the dissipation behavior of water-extractable concentrations in soils, influenced by time-dependent sorption; and (iii) the effects of OC quality, such as the chemical composition of OC and the presence of $\mathrm{BC}$, on $K_{\mathrm{oc}}$ variability.

\section{Plant Uptake of the Extractable Pesticides in Soils ${ }^{25)}$}

\subsection{Relationships between pesticide concentrations in komatsuna and the extractable concentrations in soils}

The relationship between pesticide concentrations in komatsuna shoots and the extractable concentrations in soils, which were quantified by a sequential soil extraction with water and acetone, was investigated in 8 pesticides and 4 soils.

The residue concentrations of a given pesticide in komatsuna shoots, which were cultivated on pesticide-applied soil, varied among test soils (Fig. 1), and the coefficients of variation (CVs) of the concentrations for a given pesticide ranged from $17.6 \%$ for metalaxyl to $123.5 \%$ for thiacloprid. Overall, the shoot concentrations for two andosols, which have relatively high OC content and strong sorption of pesticides, were lower than those for non-andosoils. On the other hand, for metalaxyl and fosthiazate, which have very weak sorption by soils, the variability of concentrations in komatsuna shoots was especially low; the CVs of these pesticides were less than $30 \%$. Therefore, one reason for the high variability of the shoot concentrations is considered to be the differences in the soil sorption strength.

The relationship between the pesticide concentrations in komatsuna shoots and the extractable concentrations in soils was analyzed using linear regression analysis (Table 1). Although the coefficient of determination $\left(R^{2}\right)$ values differed according to the type of pesticide, the concentrations of pesticides, except for metalaxyl and fosthiazate, in komatsuna shoots were more strongly correlated with the concentrations of water-extractable pesticides $\left(C_{\mathrm{W}}\right)$ than those of total extractable pesticides $\left(C_{\mathrm{T}}\right)$. Therefore, this result implies that the $C_{\mathrm{W}}$ values, which are influenced by the soil sorption of pesticides, can be available for evaluating the phytoavailability of residual pesticides in soils and

Table 1. Linear regression analysis ${ }^{a}$ between pesticide concentrations in komatsuna shoots and extractable concentrations ${ }^{b}$ in four soils

\begin{tabular}{lccc}
\hline \multirow{2}{*}{ Compound } & No. soils & \multicolumn{2}{c}{$R^{2}$} \\
\cline { 3 - 4 } & & Water-extracts & Total-extracts \\
\hline Imidacloprid & 4 & 0.733 & 0.332 \\
Clothianidin & 4 & $0.918^{c}$ & 0.462 \\
Thiacloprid & 4 & $0.975^{d)}$ & 0.123 \\
Metalaxyl & 4 & 0.648 & $0.844^{c}$ \\
Fosthiazate & 4 & 0.466 & 0.732 \\
Flutolanil & 4 & 0.566 & 0.543 \\
Procymidone & 4 & 0.714 & 0.489 \\
Tetraconazole & 4 & $0.976^{d)}$ & 0.334 \\
\hline
\end{tabular}

a) Regression lines were calculated using data containing the point $(0,0)$, i.e., $n=5{ }^{b)}$ Mean values during the period between sowing and harvest. ${ }^{c)}$ Significant at $p<0.05 ;{ }^{d)}$ significant at $p<0.01$. 

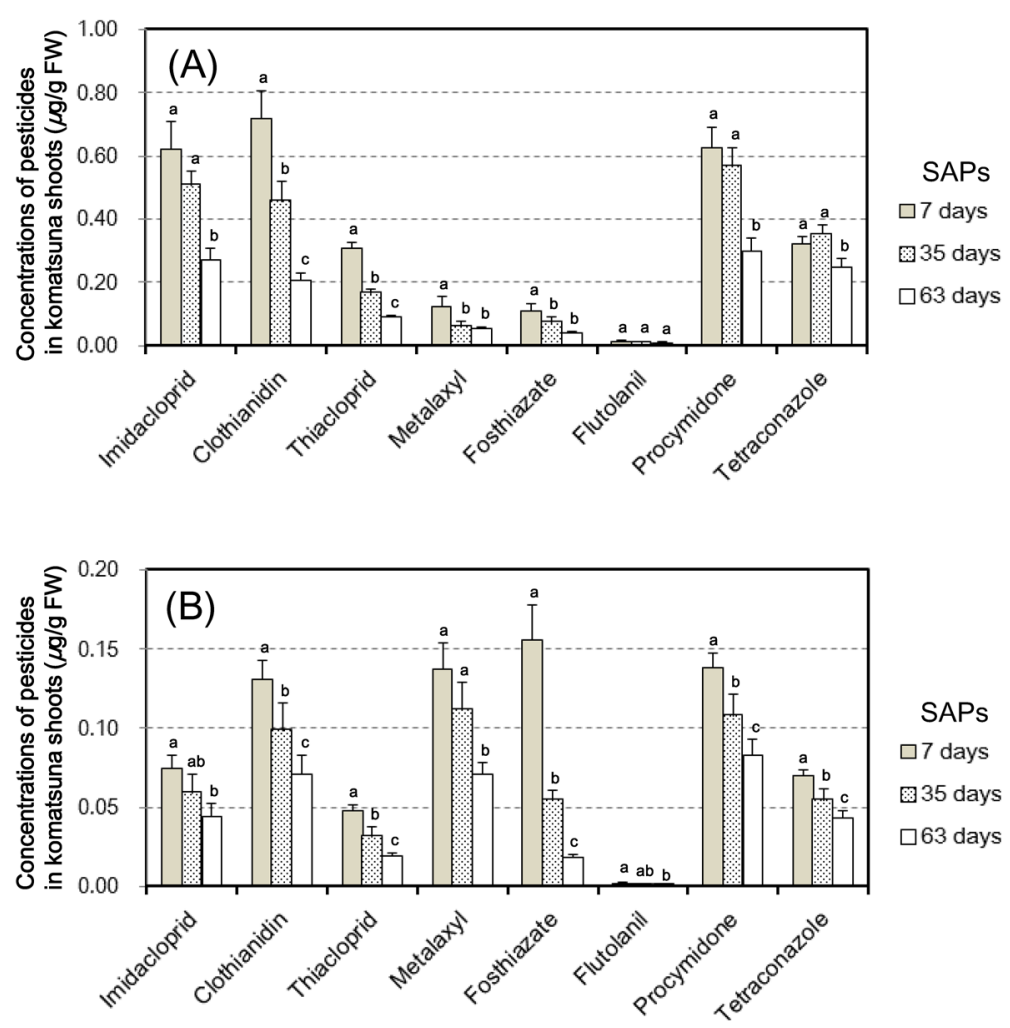

Fig. 2. Effect of soil-aging periods (SAPs) on residue concentrations of pesticides in komatsuna shoots cultivated in a gray lowland soil (A) and an andosol (B). Columns with the same letter are not significantly different at $p<0.05$, according to ANOVA-protected Tukey's multiple range testing. Error bars indicate standard deviations $(n=4)$. Reprinted from Ref. 25.

estimating the residual concentrations in succeeding crops.

\subsection{Influence of soil-aging periods (SAPs) on pesticide concentrations in komatsuna}

The effects of soil-aging periods (SAPs) — the periods between pesticide applications and sowing-on pesticide concentrations in komatsuna shoots were investigated. Figure 2 shows the relationships between the shoot concentrations and three different SAPs-7-day, 35-day, and 63-day-for a gray lowland soil and an andosol. Shoot concentrations decreased with increasing SAPs. The ratios of the residue concentrations in komatsuna shoots cultivated in soils with a 63-day SAP as compared to those with a 7 -day SAP were $29-90 \%($ mean $=50 \%)$ and $12-69 \%$ $($ mean $=51 \%)$ in a gray lowland soil and an andosol, respectively. As a result of correlation analysis between pesticide concentrations in komatsuna shoots cultivated in soils with three different SAPs and the extractable concentrations in these two soils, the $R^{2}$ values of the regression lines calculated using the $C_{\mathrm{W}}$ values were higher than those using the $C_{\mathrm{T}}$ values, except for metalaxyl and fosthiazate. These results suggest that estimating pesticide concentrations in crops based on the $C_{\mathrm{W}}$ values and setting appropriate SAPs lead to preventing the production of succeeding crops contaminated with pesticide residues in the soil.

\section{Estimation of the Dissipation Behavior of Phytoavailable Pesticides in Soils ${ }^{26)}$}

\subsection{Dissipation behavior of extractable pesticides in soils}

To estimate the pesticide concentrations in succeeding crops contaminated by pesticide residues in soils, the dissipation behavior of water-extractable pesticides, i.e., phytoavailable pesticides, in soils should be investigated. Therefore, a laboratory

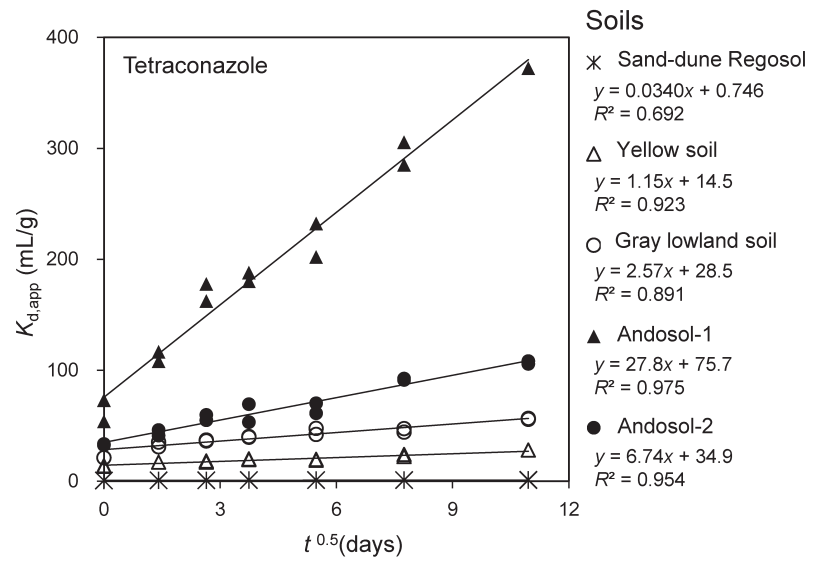

Fig. 3. Time-dependent changes in apparent sorption coefficient $\left(K_{\mathrm{d} \text {,app }}\right)$ of tetraconazole. Reprinted with permission from Ref. 26. Copyright (2016) American Chemical Society. 
incubation study using 27 pesticides and 5 Japanese soils was conducted. The soil samples were sequentially extracted using water and acetone, and the $C_{\mathrm{W}}$ and $C_{\mathrm{T}}$ values were quantified.

The dissipation behavior in soils was clearly different between water- and total extractable pesticides. That is, the $\mathrm{DT}_{50}$, which is the time taken for a $50 \%$ decrease, of $C_{\mathrm{W}}$ was shorter than that of $C_{\mathrm{T}}$ for all test soils except a sand-dune regosol having an extremely low OC content $(<0.1 \%)$, and the dissipation of $C_{\mathrm{W}}$ was better fitted by biphasic models as compared with a single first-order (SFO) model applied to the dissipation of $C_{\mathrm{T}}$. Furthermore, the dissipation rate of $C_{\mathrm{W}}$ varied depending on the OC content of soils, and the dissipation of $C_{\mathrm{W}}$ in two andosols having high $\mathrm{OC}$ contents and high pesticide sorption ability was faster than that of other soils. Therefore, it is possible that the sorption of pesticides to OCs affects the dissipation of $C_{\mathrm{W}}$.

\subsection{Time-dependent sorption of pesticides in soils}

The previous studies ${ }^{15-24)}$ reported that the apparent sorption coefficients $\left(K_{\mathrm{d} \text {,app }}, \mathrm{mL} / \mathrm{g}\right)$, which are calculated by taking the dissipation of $C_{\mathrm{T}}$ and $C_{\mathrm{W}}$ over time into account, of many pesticides increased with increasing incubation time. According to previous reports, ${ }^{19,21,23)}$ changes in $K_{\mathrm{d}, \text { app }}$ over time could be represented as

$$
K_{\mathrm{d}, \mathrm{app}}=a+b t^{0.5}
$$

where $a$ and $b$ are empirical parameters, and $t$ is the time of incubation. Figure 3 shows an example of tetraconazole to illustrate time-dependent changes in sorption. $K_{\mathrm{d} \text { app }}$ values were strongly correlated with the square root of time, and the $a$ and $b$ values were relatively high with the condition that the OC content of soils and the octanol-water partition coefficients $\left(\log P_{\text {ow }}\right)$ of pesticides were both high.

Parameters $a$ and $b$ play an important role in the prediction of time-dependent changes in $K_{\mathrm{d} \text {,app }}$ values. The $a$ values, which represent $K_{\mathrm{d} \text {,app }}$ at time $t=0$, were approximated as the measured $K_{\mathrm{d} \text {,app }}$ values of the 0 -day incubation sample $\left(K_{\mathrm{d} \text {,app }}\left(t_{0}\right)\right)$. Moreover, the $b$ values were proportional to the $K_{\mathrm{d} \text {,app }}\left(t_{0}\right)$ values, as

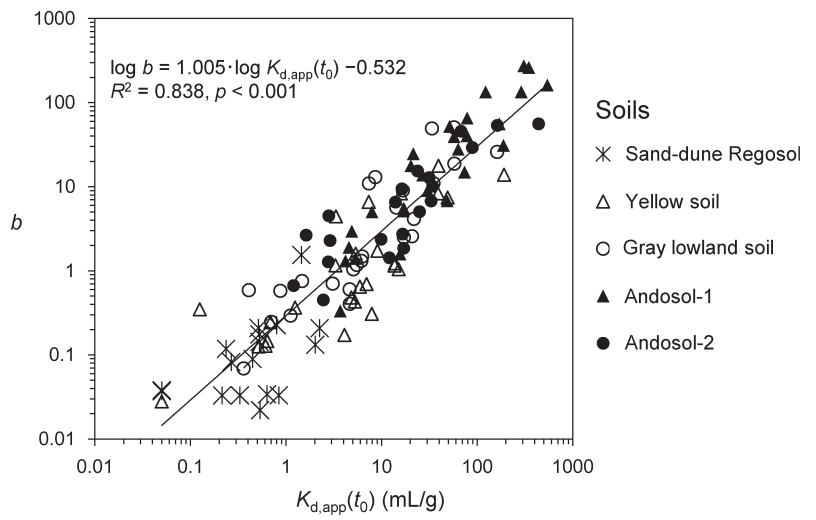

Fig. 4. Fitted parameter $b$ for Eq. 1 plotted as function of apparent sorption coefficient at 0 -day incubation $\left(K_{\mathrm{d} \text {,app }}\left(t_{0}\right)\right)$. Reprinted with permission from Ref. 26. Copyright (2016) American Chemical Society.

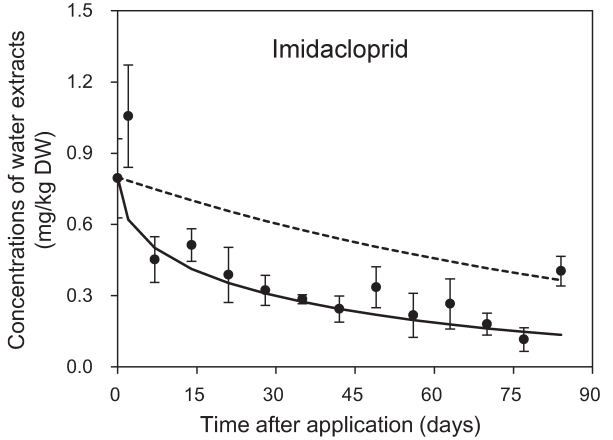

Fig. 5. Comparisons between predicted and measured concentrations of water extracts from a field soil. (-) measured values, $(-)$ values predicted using apparent sorption coefficients calculated by Eq. 2 and (----) values predicted using apparent sorption coefficient at 0 -day incubation. Error bars indicate the standard error. Reprinted with permission from Ref. 26. Copyright (2016) American Chemical Society.

shown in Fig. 4. This result suggests that parameter $b$ can be estimated using the $K_{\mathrm{d} \text {,app }}\left(t_{0}\right)$ values. Therefore, the empirical relationships between $K_{\mathrm{d} \text {,app }}\left(t_{0}\right)$ and the parameters of Eq. 1 ( $a$ and $b$ ) allow us to rewrite Eq. 1 by using $K_{\text {d,app }}\left(t_{0}\right)$ as

$$
K_{\mathrm{d}, \mathrm{app}}=K_{\mathrm{d}, \mathrm{app}}\left(t_{0}\right)+0.294 K_{\mathrm{d}, \mathrm{app}}\left(t_{0}\right)^{1.005} t^{0.5}
$$

Thus, it appears possible to estimate time-dependent changes in the $K_{\mathrm{d} \text {,app }}$ values using the $K_{\mathrm{d} \text {,app }}\left(t_{0}\right)$ values.

\subsection{Predicting the dissipation of water-extractable pesticides in a field soil}

As described in Section 2.1., the dissipation behaviors of $C_{\mathrm{W}}$ and $C_{\mathrm{T}}$ differed. It was considered that the time-dependent increase in soil sorption affected the dissipation of $C_{\mathrm{W}}$. Therefore, the dissipation of $C_{\mathrm{W}}$ was predicted by compensating for the dissipation of $C_{\mathrm{T}}$ using the time-dependent $K_{\mathrm{d} \text {,app }}$ according to the following equation:

$$
C_{\mathrm{W}}=C_{\mathrm{T}} \frac{r}{\left(r+K_{\mathrm{d}, \mathrm{app}}\right)}
$$

where $C_{\mathrm{T}}$ was calculated using the SFO model, and $r$ is the ratio of the solution to the soil $(\mathrm{mL} / \mathrm{g})$ of water extraction. The $K_{\mathrm{d} \text {,app }}$ calculated by Eq. 2 and $K_{\text {d,app }}\left(t_{0}\right)$ were used in Eq. 3 with and without consideration of time-dependent sorption, respectively. Figure 5 shows an example of imidacloprid for a comparison of the measured $C_{\mathrm{W}}$ and the predicted $C_{\mathrm{W}}$ values with and without consideration of the time-dependent increase in $K_{\mathrm{d} \text {,app. }}$. In the case of pesticides having high $K_{\mathrm{d} \text {,app }}\left(t_{0}\right)$ values $(>6 \mathrm{~mL} / \mathrm{g})$, such as clothianidin, imidacloprid, and flutolanil, the differences between the values predicted using the $K_{\mathrm{d} \text {,app }}\left(t_{0}\right)$ values and the measured values were large. In contrast, the $C_{\mathrm{W}}$ values predicted using $K_{\text {d,app }}$ estimated by Eq. 2 showed good agreement with the $C_{\mathrm{W}}$ values measured.

These results indicated that the dissipation rate of $C_{\mathrm{W}}$ was higher than that of $C_{\mathrm{T}}$, and the time-dependent increase in $K_{\mathrm{d} \text {,app }}$ affected the difference in the dissipation rate between $C_{\mathrm{W}}$ and 
Table 2. Coefficients of variation $(\mathrm{CV})$ of $K_{\mathrm{oc}}$ values and correlation coefficients $(r)$ between the $\log K_{\mathrm{oc}}$ values of pesticides and proportions of aromatic carbon in soils

\begin{tabular}{lccc}
\hline \multicolumn{1}{c}{ Compound } & No. soils & CV $(\%)$ of $K_{\text {oc values }}$ & $r$ \\
\hline Imidacloprid & 7 & 124.9 & $0.93^{a)}$ \\
Dimethoate & 4 & 82.4 & 0.60 \\
Clothianidin & 7 & 113.8 & $0.97^{a)}$ \\
Thiacloprid & 7 & 115.4 & $0.94^{a)}$ \\
Metalaxyl & 6 & 70.2 & 0.28 \\
Fosthiazate & 6 & 46.9 & 0.07 \\
Methidathion & 7 & 93.8 & $0.98^{a)}$ \\
Fenobucarb & 5 & 92.2 & 0.76 \\
Flutolanil & 7 & 65.9 & 0.39 \\
Procymidone & 7 & 84.9 & 0.57 \\
Fenitrothion & 7 & 76.3 & 0.75 \\
Tetraconazole & 7 & 50.2 & 0.30 \\
Chloroneb & 7 & 117.0 & $0.89^{a)}$ \\
Diazinon & 7 & 48.7 & -0.40 \\
Cadusafos & 7 & 59.9 & 0.40 \\
Tolclofos-methyl & 7 & 45.8 & 0.26 \\
Tetradifon & 7 & 29.5 & -0.36 \\
\hline
\end{tabular}

a) Significant at $p<0.01$.

$C_{\mathrm{T}}$. Therefore, it is possible that the dissipation of $C_{\mathrm{W}}$, which is important for evaluating the phytoavailability, is predicted by correcting the dissipation of $C_{\mathrm{T}}$ by time-dependent $K_{\mathrm{d} \text { app }}$.

\section{Factors Influencing the Sorption of Pesticides to Soils ${ }^{27)}$}

\subsection{Variability of the OC-normalized sorption coefficient $\left(\mathrm{K}_{o c}\right)$}

As described in Section 2 above, to estimate the dissipation behavior of water-extractable pesticides-phytoavailable pesticides - in soils, the $K_{\mathrm{d} \text {,app }}\left(t_{0}\right)$ should be measured. However, when the $K_{\mathrm{d} \text { app }}\left(t_{0}\right)$ was compared to the $K_{\mathrm{d}}$ measured by OECD test guidelines, ${ }^{14)}$ these values were approximately equal. Therefore, the elucidation of factors influencing the $K_{\mathrm{d}}$ variability and the estimation of $K_{d}$ values using these factors leads to predicting the dissipation of phytoavailable pesticides in soils. The $K_{\mathrm{d}}$ values of 17 pesticides in 7 typical Japanese soils were measured in accordance with OECD test guidelines, and the relationships between the $K_{\mathrm{d}}$ values and the properties of pesticides and soils were investigated.

Previous sorption studies using foreign soils suggested that the variability of the $K_{d}$ values of nonionic pesticides is strongly affected by the OC content in soil and the hydrophobicity, i.e., the $\log P_{\text {ow }}$, of pesticides. ${ }^{4)}$ Therefore, $K_{\mathrm{oc}}$ values are known to have low variability among soils and can be estimated using the $\log P_{\text {ow }}$ of pesticides. However, the $K_{\mathrm{oc}}$ values measured in 7 Japanese soils of a given pesticide exhibited high variability among soils, i.e., the CVs of the $K_{\mathrm{oc}}$ values of a pesticide ranged from 29.5 to $125 \%$ and the CVs of the $K_{\text {oc }}$ values for 11 pesticides exceeded $60 \%$ (Table 2). Wauchope et al. ${ }^{4}$ reviewed the variability of the $K_{\mathrm{oc}}$ values in the literature mainly for foreign soils and re- ported that the typical CVs of the $K_{\mathrm{oc}}$ values of a given pesticide range from 40 to $60 \%$. This result implies that the variability of the $K_{\mathrm{oc}}$ values in Japanese soils was higher than that in foreign soils. On the other hand, the correlation between the $\log K_{\mathrm{oc}}$ and $\log P_{\text {ow }}$ was poor, especially for two test andosols because the $K_{\mathrm{oc}}$ values varied depending on the molecular structures of pesticides, i.e., the $K_{\mathrm{oc}}$ values of the aromatic compounds were larger than those of non-aromatic compounds. These results imply that the sorption of pesticides to soils is affected not only by hydrophobic interaction but also by interactions involving aromatic rings such as $\pi-\pi$ interaction.

\subsection{Effect of $O C$ quality on the $\mathrm{K}_{o c}$ variability of pesticides}

Solid-state ${ }^{13} \mathrm{C}$ nuclear magnetic resonance was performed to elucidate the effect of $\mathrm{OC}$ quality, i.e., the relative proportions of each carbon type (alkyl carbon, $\mathrm{O}$-alkyl carbon, aromatic carbon, and carboxyl carbon), on $K_{\mathrm{oc}}$ variability. The proportion of aromatic carbons, which correspond to lignin, tannin, BC, and so on, exhibited the greatest variability among test soils, ranging from 12.6 to $41.3 \%$. Two test andosols in particular exhibited greater proportions of aromatic carbon than did other soils. In addition, the $K_{\mathrm{oc}}$ values of almost all pesticides for an andosol, which had the highest aromatic carbon content, were substantially higher than those for other soils. Focusing on the relationship between the $K_{\mathrm{oc}}$ and the proportion of aromatic carbon, the $\log K_{\mathrm{oc}}$ values for several pesticides were positively correlated with the proportions of aromatic carbon. The correlation coefficients $(r)$ increased with the increasing CVs of the $K_{\mathrm{oc}}$ values for a given pesticide (Table 2 ).

The sorption of organic chemicals to BC is influenced by hydrophobicity, the presence of aromatic rings, and the planarity of the molecular structure. ${ }^{28-30)}$ In other words, given the same $\log P_{\text {ow }}$ value, the sorption of planar aromatics to $\mathrm{BC}$ is higher

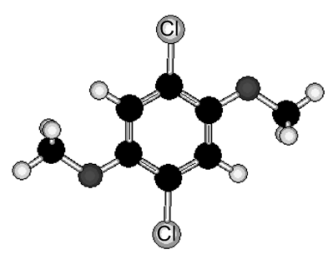

Chloroneb

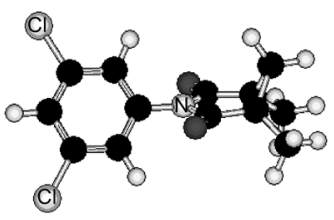

Procymidone

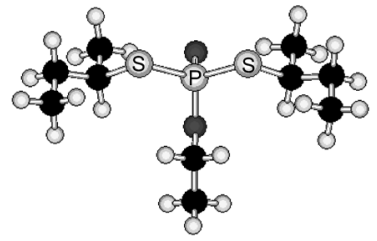

Cadusafos

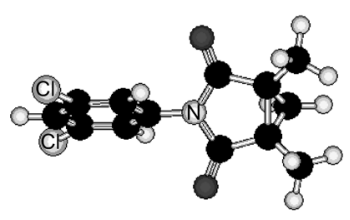

Fig. 6. Molecular structures of pesticides optimized using the PM7 semi-empirical Hamiltonian function in the MOPAC 2012 package. ${ }^{31)}$ Reprinted from Ref. 27. $\bigcirc-$ Hydrogen atom, - oxygen atom, carbon atom, (P)-phosphorus atom, (C)-chlorine atom, (N)-nitrogen atom, (S)-sulfur atom. 

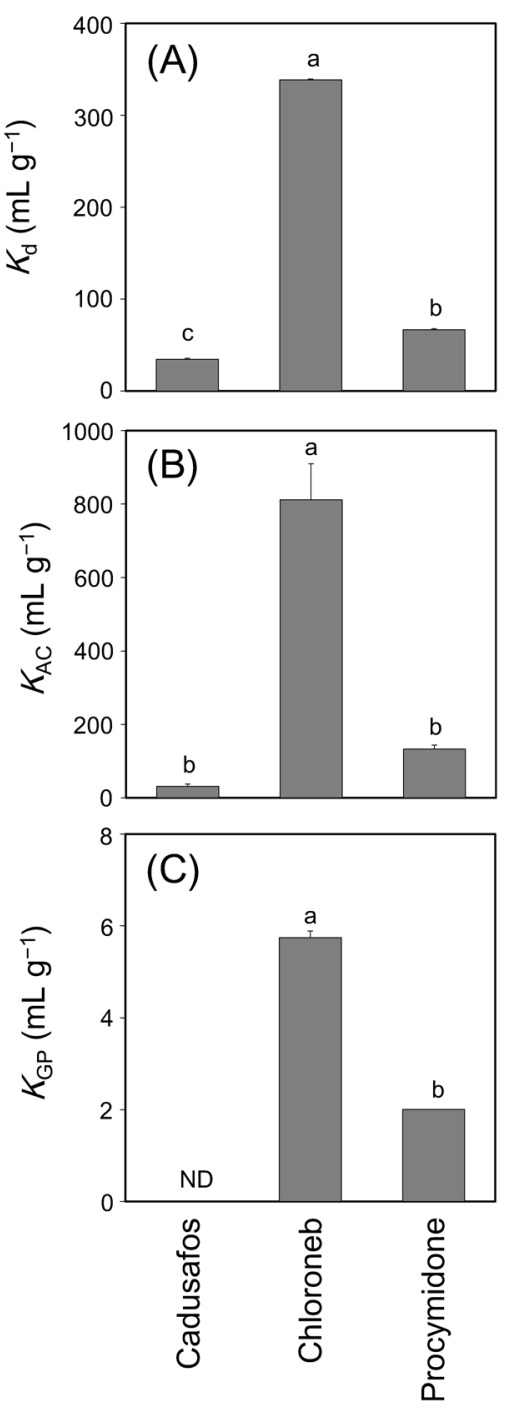

Fig. 7. (A) Soil-water distribution coefficients $\left(K_{\mathrm{d}}\right)$ for an andosol, (B) activated carbon-acetone distribution coefficients $\left(K_{\mathrm{AC}}\right),(\mathrm{C})$ graphiteacetone distribution coefficients $\left(K_{\mathrm{GP}}\right)$. Columns with the same letter are not significantly different at $p<0.05$ by ANOVA with Tukey's multiple range test for (A) and (B) and unpaired 2-sided $t$-tests for (C). Error bars indicate standard deviations $(n=3)$. ND: not detectable. Reprinted from Ref. 27.

than that of aliphatics or non-planar aromatics. For an andosol, in which the highest aromatic carbon content may be involved in the abundance of BC, the $K_{\mathrm{d}}$ values of chloroneb (a planar aromatic) were higher than those of cadusafos (an aliphatic) and procymidone (a non-planar aromatic with a torsional structure) (Figs. 6 and 7); this is in spite of the fact that the $\log P_{\text {ow }}$ of chloroneb is nearly the same as that of procymidone and lower than that of cadusafos. Similar to the results for the andosol, the sorption of chloroneb to activated carbon and graphite carbon as a model substance of $\mathrm{BC}$ was significantly greater than that of cadusafos or procymidone (Fig. 7). Therefore, it is possible that the $\mathrm{BC}$ contained in the andosol greatly affects the sorption behavior of pesticides.

\section{Concluding Remarks}

To develop a method for estimating the pesticide concentrations in succeeding crops on the predicted concentrations in soils, the dissipation behavior of phytoavailable pesticides in soils should be evaluated. The present study reveals that (i) the concentrations of water-extractable pesticides in soil can be used to evaluate the phytoavailability of pesticides in soils and (ii) the dissipation behavior of water-extractable pesticides in soils is estimated using the dissipation rates of total extractable pesticides in soils and the time-dependent $K_{\text {d,app}}$, which is predicted on the basis of the $K_{\mathrm{d}}$ measured in accordance with OECD test guidelines. Furthermore, the present study suggests that the $K_{\mathrm{d}}$ can be more precisely predicted by considering not only the OC content in soil and the hydrophobicity of pesticides but also the BC content in soil and the molecular structure of pesticides. Regrettably, no standard method for quantifying the $\mathrm{BC}$ in soils has been established. Therefore, further studies are needed to isolate and quantify the BC in soils.

\section{Acknowledgements}

I would like to thank the Pesticide Science Society of Japan, which gave me an honored award. This research was mainly conducted at the Institute for Agro-Environmental Sciences, NARO (formerly the National Institute for Agro-Environmental Sciences: NIAES). I acknowledge the members of the NIAES: Dr. Yasuhiro Yogo (former Research Coordinator), Dr. Takashi Otani (former Director of the Organochemicals Division), Dr. Nobuyasu Seike, Dr. Keiya Inao, and Dr. Sayuri Namiki. I am grateful to Dr. Satoru Ishihara (Ministry of Agriculture, Forestry and Fisheries, Japan), Dr. Takashi Iwafune (Food and Agricultural Materials Inspection Center), and Professor Hirozumi Watanabe (Tokyo University of Agriculture and Technology) for their valuable suggestions. This research was supported by the Environmental Research and Technology Development Fund (5-1302) of the Ministry of the Environment, Japan. The opinions expressed in this paper are solely those of the author.

\section{References}

1) S. Hori: Foods Food Ingredients J. Jpn. 212, 496-508 (2007) (in Japanese with English summary.)

2) H. Sugiyama, K. Komamiya and K. Kobayashi: Weed Res. Japan 35, 122-128 (1990) (in Japanese with English summary).

3) H. Sugiyama and K. Kobayashi: Weed Res. Japan 38, 300-306 (1993).

4) R. D. Wauchope, S. Yeh, J. B. Linders, R. Kloskowski, K. Tanaka, B. Rubin, A. Katayama, W. Kördel, Z. Gerstl, M. Lane and J. B. Unsworth: Pest Manag. Sci. 58, 419-445 (2002).

5) Z. Gerstl: J. Contam. Hydrol. 6, 357-375 (1990).

6) P. J. Mitchell and M. J. Simpson: Environ. Sci. Technol. 47, 412-419 (2013).

7) R. Ahmad, R. S. Kookana, A. M. Alston and J. O. Skjemstad: Environ. Sci. Technol. 35, 878-884 (2001).

8) A. G. Ahangara, R. J. Smernika, R. S. Kookanaa and D. J. Chittleborougha: Chemosphere 70, 1153-1160 (2008).

9) A. Golchin, P. Clarke, J. A. Baldock, T. Higashi, J. O. Skjemstad and J. M. Oades: Geoderma 76, 155-174 (1997).

10) J. A. González-Pérez, F. J. González-Vila, G. Almendros and H. Knicker: Environ. Int. 30, 855-870 (2004).

11) H. Shindo, T. Honna, S. Yamamoto and H. Honma: Org. Geochem. 
35, 235-241 (2004).

12) M. J. Simpson and P. G. Hatcher: Org. Geochem. 35, 923-935 (2004).

13) V. A. Loganathan, Y. Feng, G. D. Sheng and T. P. Clement: Soil Sci. Soc. Am. J. 73, 967-974 (2009).

14) OECD: “Guidelines for Testing of Chemicals," Section 1 (106), OECD, Paris, 2000.

15) L. Cox, W. C. Koskinen and P. Y. Yen: Soil Sci. Soc. Am. J. 62, 342-347 (1998).

16) M. Oi: J. Agric. Food Chem. 47, 327-332 (1999).

17) W. C. Koskinen, L. Cox and P. Y. Yen: Biol. Fertil. Soils 33, 546-550 (2001).

18) V. Laabs and W. Amelung: J. Agric. Food Chem. 53, 7184-7192 (2005).

19) C. Beigel, E. Barriuso and L. Di Pietro: J. Environ. Qual. 26, 1503 1510 (1997).

20) C. Roy, P. Gaillardon and F. Montfort: Pest Manag. Sci. 56, 795-803 (2000).

21) A. Walker: Weed Res. 27, 143-152 (1987).
22) J. B. Regitano, W. C. Koskinen and M. J. Sadowsky: J. Agric. Food Chem. 54, 1373-1379 (2006).

23) X. Louchart and M. Voltz: Environ. Sci. Technol. 41, 1137-1144 (2007).

24) J. B. Regitano and W. C. Koskinen: J. Agric. Food Chem. 56, 58015805 (2008).

25) Y. Motoki, T. Iwafune, N. Seike, T. Otani and Y. Akiyama: J. Pestic. Sci. 40, 175-183 (2015).

26) Y. Motoki, T. Iwafune, N. Seike, K. Inao and T. Otani: J. Agric. Food Chem. 64, 4478-4486 (2016).

27) Y. Motoki, T. Iwafune, N. Seike, T. Otani and M. Asano: J. Pestic. Sci. 39, 105-114 (2014).

28) T. D. Bucheli and O. V. Gustafsson: Chemosphere 53, 515-522 (2003).

29) A. Kawashima, M. Katayama and K. Honda: J. Environ.Chem. 19, 519-525 (2009) (in Japanese with English summary).

30) A. Sobek, N. Stamm and T. D. Bucheli: Environ. Sci. Technol. 43, 8147-8152 (2009).

31) http://openmopac.net/ (Accessed 19 March, 2020) 